\title{
A case of postpartum maternal death with COVID- 19 in the west of Iran
}

Maryam Ahmadi

Mehrangiz Zamani bonab

Sorour Akbari

Hamideh parsapour

Shohreh Alimohammadi

Ensiyeh Jenabi ( $\nabla$ en.jenabi@yahoo.com )

\section{Case Report}

Keywords: Coronavirus (COVID-19), Maternal, Death, Postpartum, Iran, Case report

Posted Date: May 7th, 2020

DOI: https://doi.org/10.21203/rs.3.rs-27256/v1

License: (9) This work is licensed under a Creative Commons Attribution 4.0 International License. Read Full License

Version of Record: A version of this preprint was published at Current Womens Health Reviews on December 30th, 2020. See the published version at https://doi.org/10.2174/1573404817999201230235109. 


\section{Abstract}

Background: There are a limited number of studies about COVID-19 during delivery and postpartum.

Case presentation: A 38-years old G3p2 woman at 35 weeks and 4 days of gestation referred with the chief complaints of dyspnea, cough, headache, and fever. Pharyngeal swab polymerase chain reaction (PCR) was negative for COVID-19; however, in chest computed tomography (CT) angiography, ground glass was observed in the basal lobe of the left lung. The infant was born via cesarean section with gestational age of 36 weeks and an Apgar score of 8/9. No infant document was found about COVID-19 and other infections in several days after delivery. The patient died eight days after the onset of symptoms due to cardiovascular collapse.

Conclusions: We reported the first death of postpartum maternal with COVID-19 and a healthy baby with no evidence of COVID-19 and gestational age of 36 weeks.

\section{Background}

2019 Novel coronavirus (COVID-19) has given rise to an outbreak of viral pneumonia in many countries since December 2019 (1). Novel coronavirus was first reported in Wuhan, Hubei province, China in December 2019 and spread to many countries (2).

Common symptoms at the beginning of COVID-19 infection in pregnant women include fever and cough, with less occurrence of myalgia, malaise, sore throat, diarrhea, and dyspnea. Also, lymphopenia is reported in laboratory tests (3).

Pregnant women are particularly susceptible to respiratory diseases, because they are at an immunosuppressive condition, and physiological adaptive changes during pregnancy can render them intolerant to hypoxia. For example, the mortality rate in the 1918 influenza pandemic was $2.6 \%$ in the overall population, while it was $37 \%$ among pregnant women (4).

Pregnant women are at high risk of developing a viral infection such as influenza-A, H1N1, SARS-CoV, MERS-CoV, and Ebola viruses. Therefore, they appear to have worse clinical outcomes including maternal mortality, spontaneous abortion, and preterm delivery compared to non-pregnant women (5). The clinical characteristics and vertical transmission potential of COVID-19 pneumonia in pregnant women are unknown. Therefore, there are rare studies on COVID-19 during delivery and postpartum. In this study, we reported the first death of postpartum maternal with COVID-19, Hamadan City, the west of Iran.

\section{Case Presentation}

On March 5, 2020, a 38-years old G3p2 woman, at 35 weeks and 4 days of gestation with two previous normal vaginal delivery referred to women's hospital in Hamadan City (west of Iran) with the chief 
complaint of dyspnea, cough, headache, and fever. She was a smoker (about 10 cigarettes a day) and had traveled three weeks ago to Qom (the first city that was polluted with COVID-19 in Iran).

On admission, the physical examination showed a body temperature of $38.5^{\circ} \mathrm{C}$, blood pressure of $110 / 70$ $\mathrm{mmHg}$, the pulse of 110 beats per minute, respiratory rate of $24 \mathrm{rpm}$, and $\mathrm{O}_{2}$ saturation of $88 \%$. The laboratory results included a leukocyte count of $9400 / u l$, red blood cell count $4.22 * 10^{6} / \mathrm{ul}$, platelet count $232 \star 10^{3} / \mathrm{ul}$, neutrophils of $79 \%$ Per $\mathrm{ml}$, lymphocytes of $19 \%$ Per $\mathrm{ml}$, monocytes of $2 \%$ Per $\mathrm{ml}$ and level creatinine $0.9 \mathrm{mg} / \mathrm{dl}$.

In lungs auscultation, reduction of pulmonary sounds was detected in basal areas of the left lung. The medical team was suspicious to Pulmunary thrombo Emboli after hydration/oxygenation and cardiologic consultation. Therefore, chest computed tomography (CT) angiography was performed and pulmonary thromboembolism was excluded, but the ground glass was observed in the basal lobe of the left lung (Fig. 1). Pharyngeal swab polymerase chain reaction (PCR) was negative for COVID-19. Fetal ultrasound revealed a normally intrauterine fetus with a cephalic presentation of about 35 weeks of gestational age.

COVID-19 antiviral therapy for the patient was started by a specialist in infectious disease using four antiviral drugs Oseltamivir Capsule (75 mg every 12 hours), Chloroquine sulfate Tablet (400 mg stat), Lopinavir and Ritonavir Tablets (400/100mg administered orally every 12 hours). Also, $\mathrm{O}_{2}$ saturation was increased from 91 to $94 \%$ with the oxygen mask. Fever sometimes reduced with Paracetamol $(500 \mathrm{mg}$ every 12 hours) prescription, but it was persistent at about 38 to $39^{\circ} \mathrm{C}$ without antipyretic therapy. The $\mathrm{O}_{2}$ saturation without the oxygen mask declined to $93 \%$.

The patient complained of dyspnea and no response was observed using the mentioned four antiviral drugs. On the second day of the admission, severe infection occurred and the low $\mathrm{O}_{2}$ saturation level proceeded. Then, the cesarean section was planned for the patient due to the severe dyspnea and the risk of intrapartum mother-to-child transmission by vaginal delivery. The cesarean section was performed with local anesthesia and $\mathrm{O}_{2}$ saturation around 91 to $94 \%$. The infant was born with gestational age of 36 weeks, an Apgar score of $8 / 9$ and birth weight of $3070 \mathrm{~g}$. The infant was examined for COVID-19 and other infections, but no symptom of infection with COVID-19 was found in the infant within several days after delivery.

After a cesarean section, COVID- 19 treatment was continued and $\mathrm{O}_{2}$ saturation was maintained at about 91 to $93 \%$ using an oxygen mask. Fever was persisted without Paracetamol. One day after cesarean, she felt less dyspnea, but cough and fever persisted. About 36 hours after the cesarean, in the early morning, she felt that her dyspnea was worsened. $\mathrm{O}_{2}$ saturation dropped to $83 \%$, and then the anesthesiologist decided to intubate the patient due to the low oxygen saturation. On March 9, 2020, she was transferred to the intensive care unit (ICU) in COVID- 19 central hospitals. The CT SCAN of lungs was conducted, which demonstrated bilateral infiltrations in several sections of the lungs (Fig. 2). After the intubation, $\mathrm{O}_{2}$ saturation was preserved around 95\%, but after 4 days (on March 13, 2020) cardiovascular collapse occurred and unfortunately she was expired after two resuscitations. 


\section{Discussion And Conclusion}

In this case study, we reported the first death of postpartum maternal with COVID-19 and a healthy baby with no evidence of COVID-19 and gestational age of 36 weeks. We understood that a negative PCR test is not a suitable method for the diagnosis of COVID-19 pneumonia. In this case, the lung CT scan showed a better diagnosis. Also, the efficiency of four anti-COVID-19 drugs was unclear in this case.

New COVID-19 has spread widely in several countries and a pandemic has occurred (3). Wong et al. reported that about $50 \%$ of pregnant women with SARS were admitted to the ICU, the mechanical ventilation was needed in $33 \%$ of pregnant women, and the mortality rate was more than $25 \%$ for these women (6). SARS-CoV-2 has more than $85 \%$ sequence similarity with $\operatorname{SARS}(7,8)$.

Wong et al. (2003) investigated 10 pregnant women with SARS in Hong Kong. They reported that SARSCoV infection could be associated with poor pregnancy outcomes such as maternal illness, spontaneous abortion, maternal death, and preterm birth (9).

There are many opinions about the severity of COVID-19 pneumonia in pregnancy (4). In this regard, according to many physicians, the ARDS pattern was very efficient. MacLaren et al. reported that using the ExtraCorporal membrane Oxygenation can save the patient (10), but this equipment was not available in our province (Hamadan, Iran). Several studies must be performed to demonstrate the effect of the COVID-19 virus on pregnancy. According to Chen et al. in China, all pregnant women with COVID-19 pneumonia showed a similar pattern of clinical characteristics to non-pregnant adult women. Nine pregnant women with COVID-19 participated in the study (3). However, the present case did not show this pattern. Smoking may be the cause of the severity and rapid progression of ARDS.

The present case provides a view of the outcome associated with COVID-19 during delivery and postpartum. Comprehensive data with high sample size from pregnant women with COVID-19 are needed to better understand the overall effect of COVID-19 on maternal and infant outcomes.

\section{Abbreviations}

PCR: Pharyngeal swab polymerase chain reaction; CT: chest computed tomography; ICU: the intensive care unit

\section{Declarations}

Acknowledgements: Not applicable.

Authors' contributions: MA and EJ were responsible for writing the manuscript. MZ, MA, HP and SA collected the patient data. The cesarean section was performed by MA. SA was responsible for reading the $\mathrm{CT}$ angiography. All authors read and approved the final manuscript.

Funding: No financial support was received for this study. 
Availability of data and materials: Not applicable.

Ethics approval and consent to participate: Not applicable.

Consent for publication: Written consent was obtained from the patient's Husband to allow the use of the patient's file information for publication.

Competing interests: The authors declare that they have no competing interests.

Author details: ${ }^{1,2,4,5}$ Clinical Research Development Unit of Fatemieh Hospital, Department of Gynecology, School of Medicine, Hamadan University of Medical Sciences, Hamadan, Iran. ${ }^{3}$ Clinical Research Development Unit of Fatemieh Hospital, Department of Gynecology, School of Medicine, Hamadan University of Medical Sciences, Hamadan, Iran. ${ }^{6}$ Autism Spectrum Disorders Research Center, Hamadan University of Medical Sciences, Hamadan, Iran.

\section{References}

1. Chan JF-W, Yuan S, Kok K-H, To KK-W, Chu H, Yang J, et al. A familial cluster of pneumonia associated with the 2019 novel coronavirus indicating person-to-person transmission: a study of a family cluster. The Lancet. 2020;395(10223):514-23.

2. Huang C, Wang Y, Li X, Ren L, Zhao J, Hu Y, et al. Clinical features of patients infected with 2019 novel coronavirus in Wuhan, China. The Lancet. 2020;395(10223):497-506.

3. Chen H, Guo J, Wang C, Luo F, Yu X, Zhang W, et al. Clinical characteristics and intrauterine vertical transmission potential of COVID-19 infection in nine pregnant women: a retrospective review of medical records. The Lancet. 2020;395(10226):809-15.

4. Gottfredsson M. The Spanish flu in Iceland 1918. Lessons in medicine and history. Laeknabladid. 2008;94(11):737-45.

5. Wang1a X, Zhou2a Z, Zhang J, Zhu F, Tang Y, Shen X, et al. A case of 2019 Novel Coronavirus in a pregnant woman with preterm delivery. 2020.

6. Wong SF, Chow KM, Leung TN, Ng WF, Ng TK, Shek CC, et al. Pregnancy and perinatal outcomes of women with severe acute respiratory syndrome. American journal of obstetrics and gynecology. 2004;191(1):292-7.

7. Schoeman D, Fielding BC. Coronavirus envelope protein: current knowledge. Virology journal. 2019;16(1):69.

8. de Wit E, van Doremalen N, Falzarano D, Munster VJ. SARS and MERS: recent insights into emerging coronaviruses. Nature Reviews Microbiology. 2016;14(8):523.

9. Wong S, Chow K, de Swiet M. Severe acute respiratory syndrome and pregnancy. BJOG: An International Journal of Obstetrics \& Gynaecology. 2003;110(7):641-2.

10. MacLaren G, Fisher D, Brodie D. Preparing for the most critically ill patients with COVID-19: the potential role of extracorporeal membrane oxygenation. Jama. 2020. 
Figures

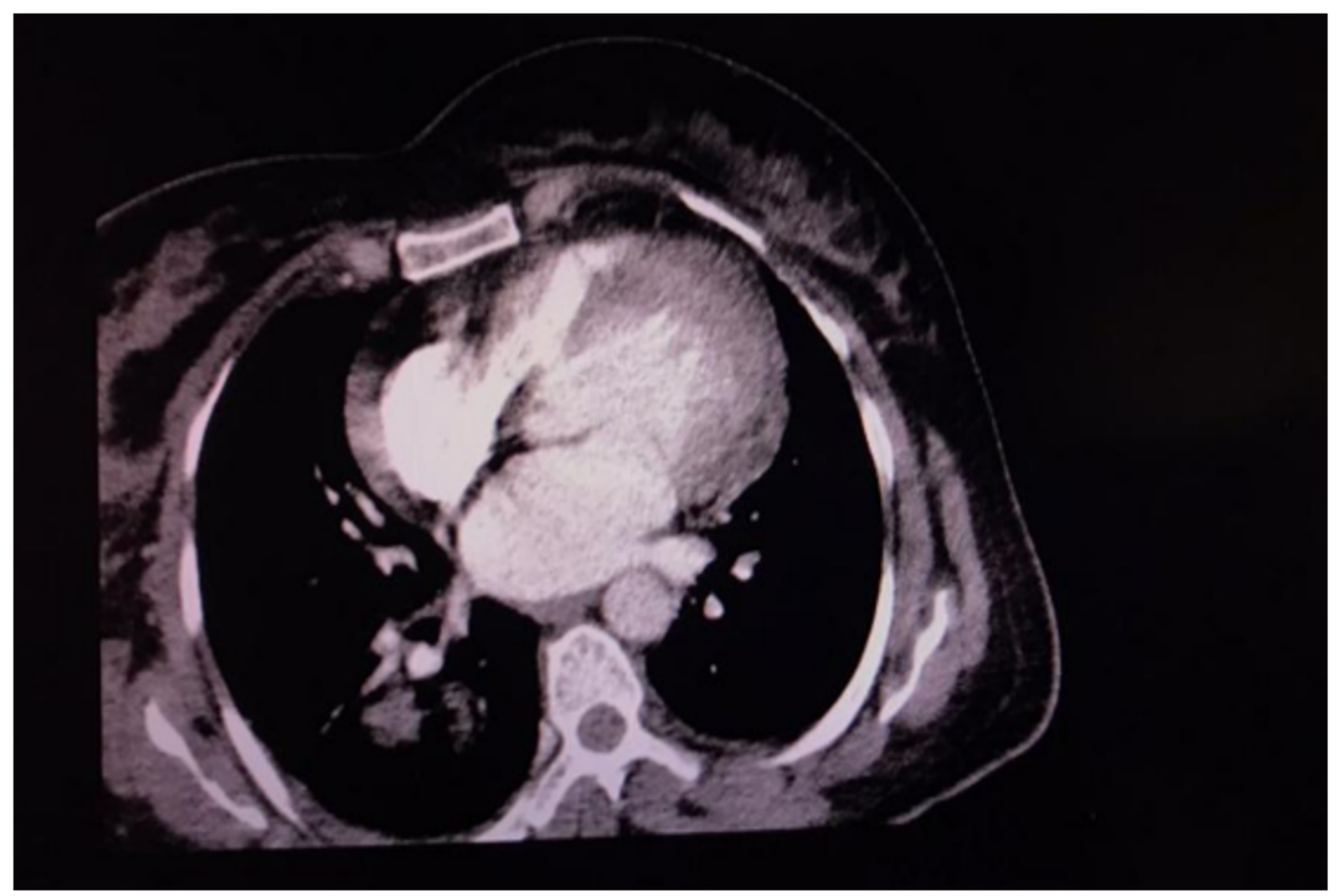

Figure 1

March 6, 2020, CT angiography of the mother. The ground glass in the basal lobe of the left lung 


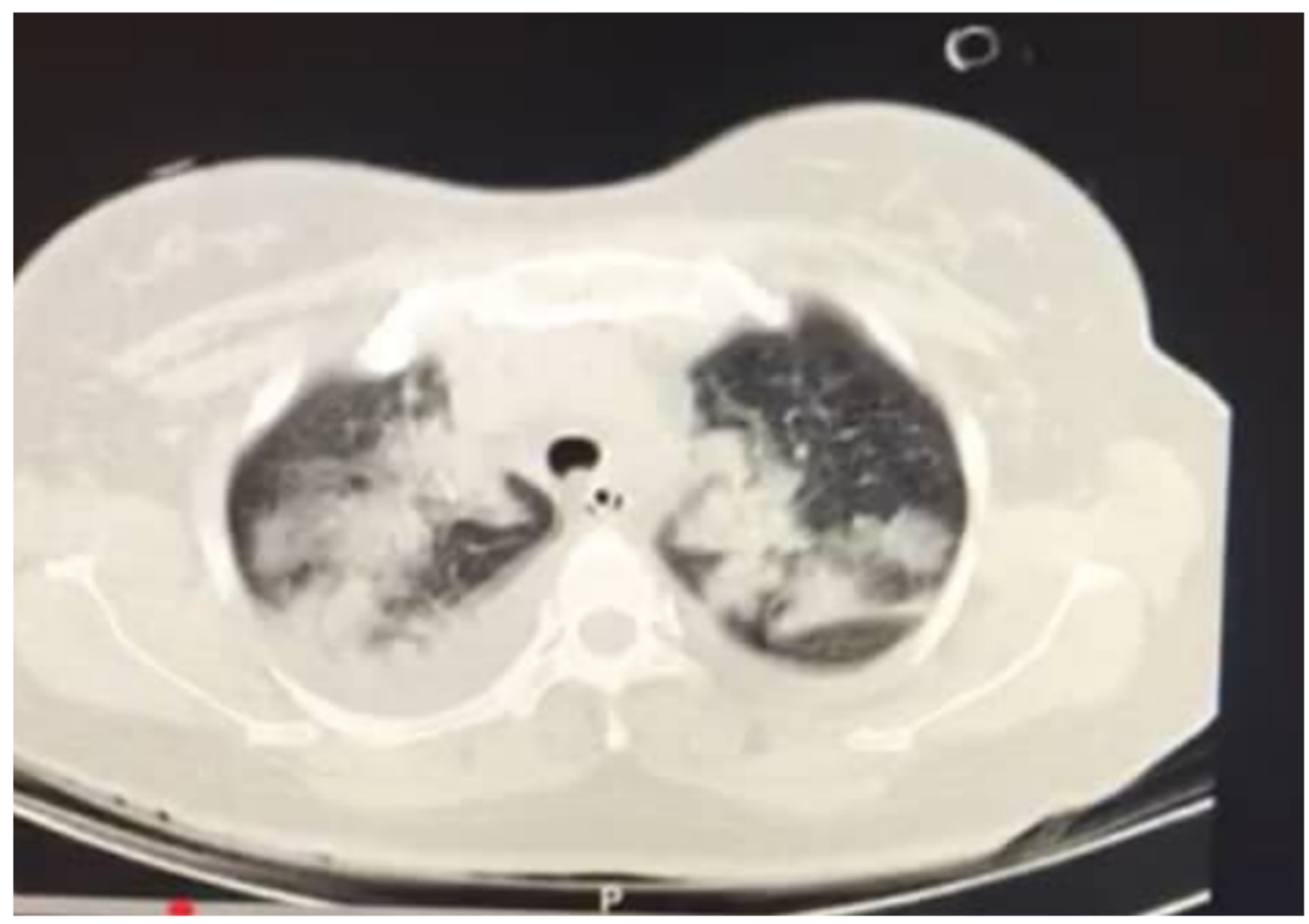

Figure 2

March 11, 2020, CT angiography of the mother. The bilateral infiltrations in several sections of the lungs 